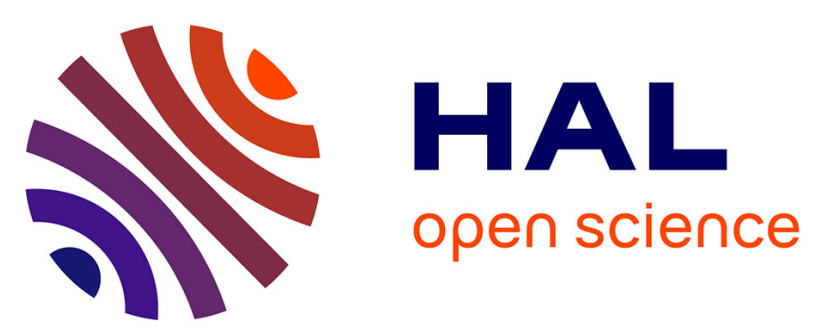

\title{
Dispersion of the nonlinear susceptibility of MoS2 and WS2 from second-harmonic scattering spectroscopy
}

Kévin Bredillet, Jérémy Riporto, Gregory T Forcherio, Jeremy R Dunklin, Jean-Pierre Wolf, Luigi Bonacina, Yannick Mugnier, Ronan Le Dantec

\section{- To cite this version:}

Kévin Bredillet, Jérémy Riporto, Gregory T Forcherio, Jeremy R Dunklin, Jean-Pierre Wolf, et al.. Dispersion of the nonlinear susceptibility of MoS2 and WS2 from second-harmonic scattering spectroscopy. Physical Review B, 2020, 102 (23), pp.235408. 10.1103/PhysRevB.102.235408 . hal03045815

\section{HAL Id: hal-03045815 https://hal.science/hal-03045815}

Submitted on 8 Dec 2020

HAL is a multi-disciplinary open access archive for the deposit and dissemination of scientific research documents, whether they are published or not. The documents may come from teaching and research institutions in France or abroad, or from public or private research centers.
L'archive ouverte pluridisciplinaire HAL, est destinée au dépôt et à la diffusion de documents scientifiques de niveau recherche, publiés ou non, émanant des établissements d'enseignement et de recherche français ou étrangers, des laboratoires publics ou privés. 


\title{
Dispersion of the nonlinear susceptibility of $\mathrm{MoS}_{\mathbf{2}}$ and $\mathrm{WS}_{2}$ from second-harmonic scattering spectroscopy
}

\author{
Kévin Bredillet, ${ }^{1}$ Jérémy Riporto, ${ }^{1,2}$ Gregory T. Forcherio $\odot,{ }^{3}$ Jeremy R. Dunklin, ${ }^{4}$ Jean-Pierre Wolf $\odot,{ }^{2}$ Luigi Bonacina $\odot,{ }^{2}$ \\ Yannick Mugnier $\odot,{ }^{1}$ and Ronan Le Dantec ${ }^{1}{ }^{1, *}$ \\ ${ }^{1}$ Univ. Savoie Mont Blanc, SYMME, F-74000 Annecy, France \\ ${ }^{2}$ Department of Applied Physics-GAP, Université de Genève, 22 Chemin de Pinchat, CH-1211 Geneva, Switzerland \\ ${ }^{3}$ Electro-Optic Technology Division, Naval Surface Warfare Center, Crane, Indiana 47522, USA \\ ${ }^{4}$ Chemistry and Nanoscience Center, National Renewable Energy Laboratory, Golden, Colorado 80401, USA
}

(Received 7 October 2020; revised 19 November 2020; accepted 23 November 2020; published 4 December 2020)

\begin{abstract}
Dispersion of the absolute second-order susceptibility of both $\mathrm{MoS}_{2}$ and $\mathrm{WS}_{2}$ is assessed on a wide spectral excitation range $(710-1300 \mathrm{~nm})$ by using second-harmonic scattering spectroscopy (SHS). SHS is an accurate ensemble measurement here applied on well-dispersed suspensions of monodisperse liquid-exfoliated nanosheets showing a high monolayer content. The as-derived, high susceptibility values shed light on the discrepancies between available literature values while evidencing resonances associated with the main excitonic transitions.
\end{abstract}

DOI: 10.1103/PhysRevB.102.235408

\section{INTRODUCTION}

Nonlinear optical properties of two-dimensional (2D) layered materials are the subject of extensive research [1]. Among 2D materials, transition metal dichalcogenides (TMDs) constitute an attractive class because their lack of inversion symmetry confers them a strong second-order nonlinear optical response [2]. Early studies [3,4] reported very high values of the quadratic susceptibility $\chi^{(2)}$, on the order of $10^{2} \mathrm{pm} / \mathrm{V}$, then leading to a significant amount of work in this area. One of the important features of TMDs is the relationship between second-harmonic generation (SHG) and the number of layers. It was demonstrated that SHG is only observed with an odd number of layers [3,4]. For even numbers, coherent superposition of the opposite second-harmonic (SH) fields generated within each layer results in cancellation of the signal at $2 \omega$. The relationship between the stacking structure and SHG has since been exploited as a tool for TMD characterizations, to probe for instance their crystalline orientation [3] and to study homo- and heterostructured multilayer structures [5]. Another notable property of TMDs stems from the strong excitonic resonances observed in 2D materials resulting in significant enhancement of the SHG emissions around the transitions [6].

Interestingly, the $\mathrm{MoS}_{2}$ quadratic susceptibility at discrete excitation wavelengths has already been estimated (Supplemental Table S1 [7]), but a large discrepancy can be found in the literature with $\chi^{(2)}$ values spanning three orders of magnitude [1]. This large discrepancy is probably due to the difficulty in measuring and modeling the SHG response from 2D monolayers. SHG microscopy is a commonly used technique to analyze the SH signal emitted by single lay-

*ronan.le-dantec@univ-smb.fr ers, but deriving accurate quantitative values is a challenging task since the absolute calibration of a SHG microscope requires careful procedures, especially on a large spectral range. The best approach is likely to compare signals coming from monolayers and a reference material, e.g., a quartz crystal, treating individual nanosheets as a nonlinear polarization sheet. However, a different model making use of a standard SHG reflection formalism is typically employed for the reference crystal $[4,8,9]$, and most studies do not consider deviations that could occur under tight optical focusing conditions with high numerical-aperture (NA) objectives. On the other hand, a direct calibration using an estimated incident intensity at the beam waist was also proposed to derive the TMD susceptibility [10-12], but this approach appears to be delicate due to very precise identification of all beam parameters that must be integrated. The effect of the substrate can also strongly modify the emitted SH signal due to additional interference contributions [12,13], and strain artifacts are known to result in SHG variations [14]. In terms of TMD materials, a few studies have highlighted the effect of crystalline quality on discrepancies between the published $\chi^{(2)}$ values between mechanically exfoliated samples and those obtained from chemical vapor deposition (CVD) [10,15]. Regarding $\mathrm{WS}_{2}$, less work has been performed $[9,12,16,17]$, but similar observations have been noted.

There exists significant interest in measuring SHG behavior of 2D TMDs with new characterization methods, associated with appropriate models, to address these knowledge gaps. Determination of the $\chi^{(2)}$ dispersion is also very fundamentally relevant in order to quantitatively study the resonances associated with the different optoelectronic transitions. The first study by Malard et al. [3] showed a significant amplification of the SHG around the $\mathrm{C}$ exciton within $\mathrm{MoS}_{2}$ layers. Several reports have since confirmed that an enhancement occurs around the three main UV-visible excitonic 
transitions, typically denoted as A, B, and C $[6,18,19]$. One can also expect that quantitative dispersion measurements will lead to a better understanding of the nonlinear optical properties of TDMs through comparison with theoretical models [20,21].

In this paper, we use second-harmonic scattering (SHS) spectroscopy to quantitatively assess the absolute, orientationaveraged second-order susceptibility of both $\mathrm{MoS}_{2}$ and $\mathrm{WS}_{2}$. SHS spectroscopy is an ensemble measurement probing under low focusing conditions a very large number of sheets within TMD suspensions. This alternative approach was initially employed to derive the susceptibility of $\mathrm{WS}_{2}$ at a single excitation wavelength of $1064 \mathrm{~nm}$ [22]. This work expands scope to a wide excitation range $(710-1300 \mathrm{~nm})$ via a femtosecond tunable laser source to assess dispersion of the orientationaveraged $\chi^{(2)}$ and to probe impacts of the excitonic resonances of $\mathrm{MoS}_{2}$ and $\mathrm{WS}_{2}$ on their $\chi^{(2)}$ dispersion magnitude. Consistency and suitability of the SHS technique are demonstrated from different liquid-exfoliated TMD suspensions consisting of freestanding, dispersed nanosheets with typical lateral dimension below $100 \mathrm{~nm}$ and a high proportion of monolayers $(42-75 \%)$.

\section{EXPERIMENTAL PROCEDURES}

Monolayer to few-layer nanosheets of $\mathrm{WS}_{2}$ and $\mathrm{MoS}_{2}$ were suspended in aqueous solutions via liquid-phase exfoliation (LPE) [23] followed by a liquid-cascade centrifugation process [24], which together offers low-cost, scalable production of low-dimensional TMDs for SHS spectroscopy. Briefly, bulk $\mathrm{WS}_{2}$ and $\mathrm{MoS}_{2}$ powders (Sigma-Aldrich) were dispersed at a concentration of $25 \mathrm{mg} / \mathrm{mL}$ into an aqueous sodium cholate surfactant solution $(6 \mathrm{mg} / \mathrm{mL}, 80 \mathrm{~mL}$ total volume $)$ and probe sonicated at $360 \mathrm{~W}$ (600 W Cole-Parmer; 60\% amplitude) immersed in an ice-water bath. After an initial purification and cleaning step [24], the TMD powder dispersions were sonicated for $7 \mathrm{~h}$ with a 5-h on-, 2-h off-cycle for exfoliation. Size separation of the exfoliated nanosheet dispersions was executed via a liquid-cascade centrifugation process previously described [24]. Generally, nanosheets trapped at higher centrifugation speeds are smaller in both thickness and lateral dimensions. Monolayer-rich $\mathrm{WS}_{2}$ and $\mathrm{MoS}_{2}$ dispersions used in this paper were trapped between 10 and $18 \mathrm{krpm}$ ( 7.5 and $24.4 \mathrm{~kg}$ ) and between 14 and $24 \mathrm{krpm}$ (14.8 and $43.4 \mathrm{~kg}$ ), respectively (Supplemental Fig. S1). Suspensions of the monolayer-rich TMD dispersions stabilized with sodium cholate are thus obtained with no observable aggregation.

Mean nanosheet layer quantity, lateral size, and monolayer concentration metrics for each $\mathrm{WS}_{2}$ and $\mathrm{MoS}_{2}$ dispersion were assessed by empirical relationships derived from transmission UV-visible metrics, as detailed extensively in Refs. [24,25]. Representative absorbance spectra for $\mathrm{WS}_{2}$ and $\mathrm{MoS}_{2}$ are shown in Fig. 1, which contain size- and thicknessdependent signatures due to electronic structure and quantum confinement effects at the nanosheet edges. Such quantitative correlation between the intensity- and wavelength-occurrence of various spectroscopy absorbance features to size metrics of these TMD dispersions at less than $10 \%$ uncertainty has been intensely explored via statistical atomic force and transmission electron microscopies (AFM and TEM, respectively)

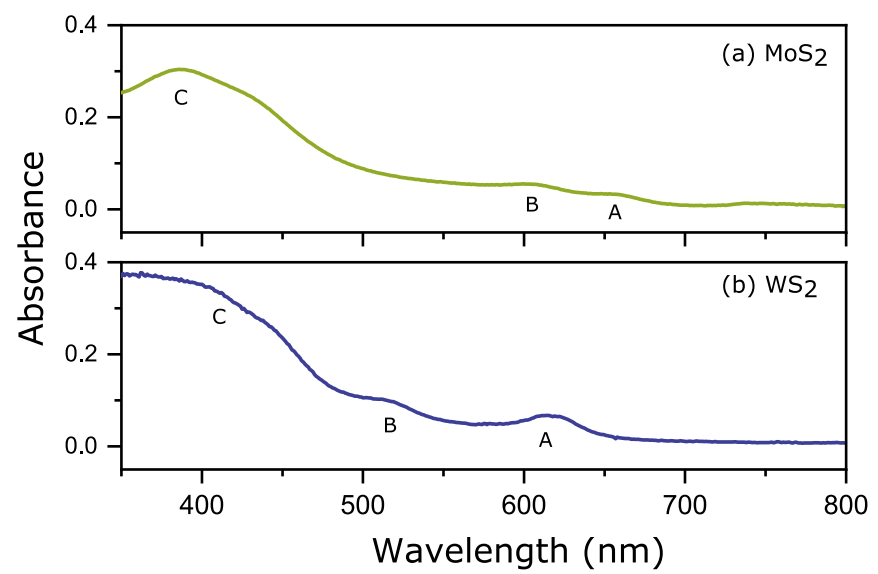

FIG. 1. Absorbance spectra of the (a) $\mathrm{MoS}_{2}$ and (b) $\mathrm{WS}_{2}$ suspensions (sample $\mathrm{w} 3$ for $\mathrm{WS}_{2}$; see Table I). The A, B, and $\mathrm{C}$ excitonic transitions are labeled.

[24-26] and dynamic light scattering (DLS) [27]. The procedure has been applied in the study of numerous $2 \mathrm{D}$ material systems [28-33]. Here, three different $\mathrm{WS}_{2}$ suspensions and one $\mathrm{MoS}_{2}$ suspension were prepared for optical characterization with measured metrics summarized in Table I.

Our approach for the measurement of the orientationaveraged second-order susceptibility is fully described in a recent publication [34], where the susceptibility dispersion of several nanomaterials such as $\mathrm{LiNbO}_{3}(\mathrm{LN}), \mathrm{BiFeO}_{3}$, and $\mathrm{ZnO}$ is quantitatively assessed in the 700-1300-nm excitation range. A two-step procedure is applied. Dispersion of the relative susceptibility is first determined by measuring for each wavelength the $\mathrm{SH}$ intensity scattered by a suspension before applying a protocol for correcting the spectral response of the setup. The absolute value of the susceptibility is then retrieved at $1064 \mathrm{~nm}$ by comparison with the well-known hyperpolarizability of para-nitroaniline (pNA) molecules dissolved in methanol [35].

Briefly, the intensity of the SH signal scattered from a colloidal suspension without any solvent contribution can be expressed as

$$
I_{2 \omega}=G N T\left\langle\beta^{2}\right\rangle I_{\omega}^{2} .
$$

$G$ is an experimental parameter, and $N$ is the number concentration of nonlinear emitters, namely, nanosheets here. $T$ is a field factor which connects the incident electric field to the

TABLE I. Sample parameters and measured susceptibilities at 1064-nm excitation. $\langle N\rangle$, mean layer quantity; mVf, monolayer volume fraction; $\langle L\rangle$, mean length; $\langle\beta\rangle$, orientation-averaged hyperpolarizability; $\chi_{x x x}^{(2)}$, bulk susceptibility.

\begin{tabular}{lcccc}
\hline \hline Sample code & w1 & $\begin{array}{l}\mathrm{WS}_{2} \\
\text { w2 }\end{array}$ & w3 & $\mathrm{MoS}_{2}$ \\
\hline$\langle N\rangle$ & 1.7 & 1.7 & 1.1 & 1.5 \\
$\mathrm{mVf}(\%)$ & 42 & 50 & 75 & 51 \\
$\langle L\rangle(\mathrm{nm})$ & 42 & 30 & 25 & 80 \\
$\langle\beta\rangle\left(10^{-26} \mathrm{esu}\right)$ & 15.8 & 5.9 & 8.0 & 20.1 \\
$\chi_{x x x}^{(2)}(\mathrm{pm} / \mathrm{V})$ & $370 \pm 50$ & $270 \pm 110$ & $530 \pm 210$ & $130 \pm 50$ \\
\hline \hline
\end{tabular}



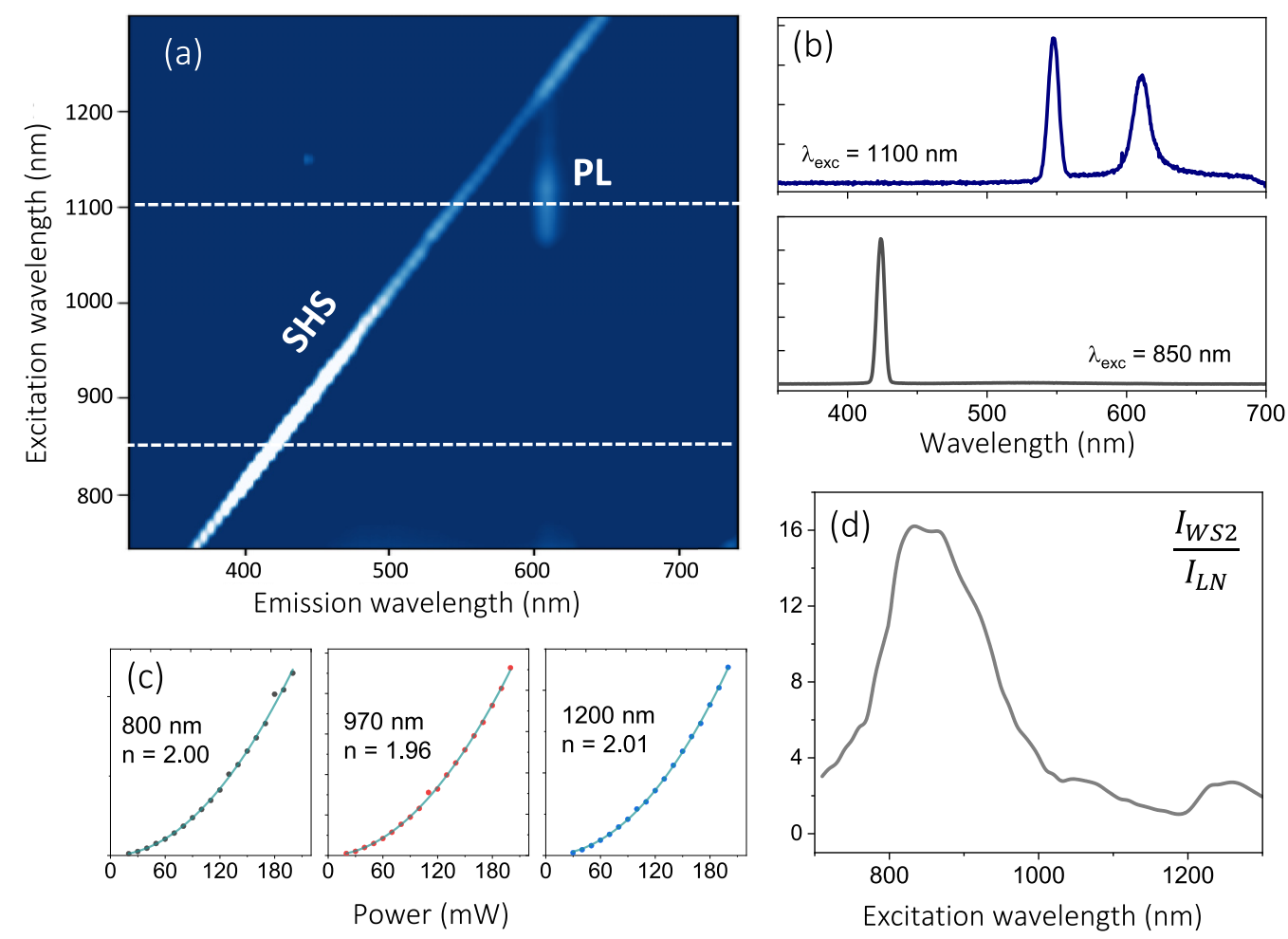

FIG. 2. (a) Surface map of scattering intensity at a given wavelength ( $x$ axis) as a function of the excitation wavelength ( $y$ axis, 10-nm wavelength increments) for a $\mathrm{WS}_{2}$ suspension (sample w2; see Table I). (b) Specific spectra at 850 and $1100 \mathrm{~nm}$ extracted from (a) showing pure SHG and both SHG and two-photon photoluminescence (PL), respectively. (c) Power dependence of SH signal at 800-, 970-, and 1200-nm excitation. The value of $n$ power resulting from the fit of the experimental curves with $A I_{\omega}^{n}$ is shown. (d) Experimental ratio of the SHS intensity $I_{\mathrm{WS}_{2}}$ and $I_{\mathrm{LN}}$ from $\mathrm{WS}_{2}$ and $\mathrm{LN}$ suspensions, respectively, according to the excitation wavelength.

inner macroscopic field within nanoparticles (see Supplemental Material [7]). For spherical nanoparticles, $T$ is calculated from the quasistatic approximation provided for a sphere, which allows us to estimate the inner field according to the refractive indices of the solvent and of the particles [36]. However, this approximation is no longer appropriate for a 2D nanosheet, and we here assume, because of the ultrathin monolayer thickness, that the incident fundamental field is not modified within the nanosheet, i.e., $T=1$ [22]. Importantly, this assumption is the same as the one commonly used to describe a nonlinear polarization sheet deposited on top of a substrate. The fundamental electric field at the substrate surface is presumably not modified by the presence of a monolayer [37].

Finally, $\left\langle\beta^{2}\right\rangle^{\frac{1}{2}}=\langle\beta\rangle$ is the effective hyperpolarizability averaged over all possible orientations. This experimental parameter is simply related to the sheet susceptibility $\left\langle\chi_{s}^{(2)}\right\rangle$ as

$$
\left\langle\chi_{s}^{(2)}\right\rangle=\frac{2\langle\beta\rangle}{S},
$$

with $S$ being the sheet surface. Dividing this sheet susceptibility by the monolayer thickness $(t=0.65 \mathrm{~nm})$ then allows derivation of the effective volume susceptibility $\left\langle\chi^{(2)}\right\rangle$ as usually reported in previous works. Comparison with the single nonzero tensor element $\chi_{x x x}^{(2)}$ retrieved in typical SHG microscopy experiments is then readily achieved since the orientation-averaging procedure leads to $\chi_{x x x}^{(2)}=-\chi_{x y y}^{(2)}=$ $-\chi_{y y x}^{(2)}=\chi_{y x y}^{(2)}=(21 / 8)^{1 / 2}\left\langle\chi^{2}\right\rangle$ for the $\mathrm{D}_{3 h}$ point-group symmetry of TMD monolayers [22].

The experimental setup described in Ref. [34] is based on a femtosecond laser (Spectra Physics Insight X3) with a wide wavelength tuning range $(680-1300 \mathrm{~nm})$. The incident beam power is controlled and kept constant over the full excitation range after careful calibration of a halfwave plate (FR600HM; Thorlabs) associated with a vertical Glan-Thomson polarizer. An achromatic lens (Newport, $f=$ $30 \mathrm{~mm}$ ) is here used to focus the incident beam into a microcuvette (CV10Q700FS; Thorlabs) containing the TMD nanosheet suspension. The optical excitation path here restrained to the $2 \mathrm{~mm}$ of the cuvette thickness allows us to limit self-defocusing that may arise for an incident beam power above $200 \mathrm{~mW}$. The SH signal collected perpendicularly to the incident beam is then focused through two fused silica lenses $(f=50 \mathrm{~mm})$ into the entrance slit of a spectrometer (Andor Shamrock 193) coupled to a CCD camera (Andor iDus 401). According to the excitation wavelength, a lowpass filter (FESH0700 or FGB37; Thorlabs) is placed in front of the spectrometer to remove any scattered signal from the fundamental beam.

\section{EXPERIMENTAL RESULTS AND DISCUSSION}

The relative susceptibility measurements performed on $\mathrm{WS}_{2}$ are summarized in Fig. 2, including emission spectra [Figs. 2(a) and 2(b)], power-dependence plots [Fig. 2(c)], 
and magnitude ratio of $\mathrm{WS}_{2}$ SHS normalized to that of $\mathrm{LN}$ [Fig. 2(d)]. The measured emission spectra consistently feature a SH signal at $2 \omega$, while a subset $(\sim 1050-1200-\mathrm{nm}$ excitation range) additionally displays a two-photon excited photoluminescence signal associated with the A exciton transition. This is illustrated verbatim in Fig. 2(b). The SHS intensity from each emission spectrum was estimated using a Gaussian fitting procedure and normalized using the SHS intensity collected from a LN suspension. Indeed, raw SHS intensity values are obviously related to the spectral response of each optical element comprising the instrumentation setup. $\mathrm{LN}$ suspension can be considered as an ideal reference for the calibration route since LN has a known susceptibility $\left\langle\chi^{(2)}\right\rangle^{\mathrm{LN}}$ which moreover slightly varies over the studied wavelength range [34]. The resulting SHS intensity ratio $I_{\mathrm{WS}_{2}} / I_{\mathrm{LN}}$, shown in Fig. 2(d), represents the instrumentation-corrected SHS signal from which the relative susceptibility is calculated as [34]

$$
\left\langle\chi^{(2)}\right\rangle_{\mathrm{Rel}}^{\mathrm{WS}_{2}} \propto\left(\frac{I_{\mathrm{WS}_{2}}}{I_{\mathrm{LN}}} T_{\mathrm{LN}}\right)^{\frac{1}{2}}\left\langle\chi^{(2)}\right\rangle^{\mathrm{LN}},
$$

where $T_{\mathrm{LN}}$ is here the field factor calculated for $\mathrm{LN}$ nanoparticles. The resulting relative susceptibility for $\mathrm{WS}_{2}$ is depicted in Supplemental Fig. S2.

As described in our previous work [34], there are some experimental parameters that require specific attention to obtain robust SHS measurements. Firstly, the SHS signal can be reabsorbed if particle suspensions are not transparent at the SH wavelength (i.e., SHS signal either falls within an absorption band of the probed sample or is strongly scattered within the suspension). To avoid this issue, sample must be diluted enough until reabsorption and scattering of the $\mathrm{SH}$ are negligible. A linearly proportional relationship between SHS signal and nanosheet number density is then observed, per Eq. (1), which is confirmed in Fig. 3 where number density values are sufficiently low $\left(<3 \times 10^{12} \mathrm{~cm}^{-3}\right)$. A second potential issue can arise with the possibility of thermal lensing that can cause variations in the excitation intensity $I_{\omega}$, which should be kept constant across the full excitation range. Herein, the laser power was fixed $(100 \mathrm{~mW})$, and an achromatic lens was used to focus the beam such that no change of the focus waist parameters should be observed. Self-defocusing through thermal lensing was noticed, however, at too high incident power exceeding $200 \mathrm{~mW}$ and within solvent absorption bands (namely, at $\lambda=970 \mathrm{~nm}$ and $\lambda=1200 \mathrm{~nm}$ for water [38]). This can lead to a change in the waist size and position and consequently a variation in the excitation intensity. Here, the absence of self-defocusing is attested from the ideal quadratic behavior observed in Fig. 2(c) for the two most sensitive excitation wavelengths ( 970 and $1200 \mathrm{~nm}$ ). After confirming the absence of these two procedural caveats, reproducible SHS intensity values over a wide excitation range were obtained. The ratio $I_{\mathrm{WS}_{2}} / I_{\mathrm{LN}}$ in Fig. 2(d) shows the relative intensity of SHS from $\mathrm{WS}_{2}$ after correcting for instrument function of the setup, where three resonances associated with the excitonic transitions of $\mathrm{WS}_{2}$ are apparent. They will be discussed in more detail later. The same experimental procedure was then applied to $\mathrm{MoS}_{2}$ suspensions, whose results are presented in Supplemental Fig. S3. For

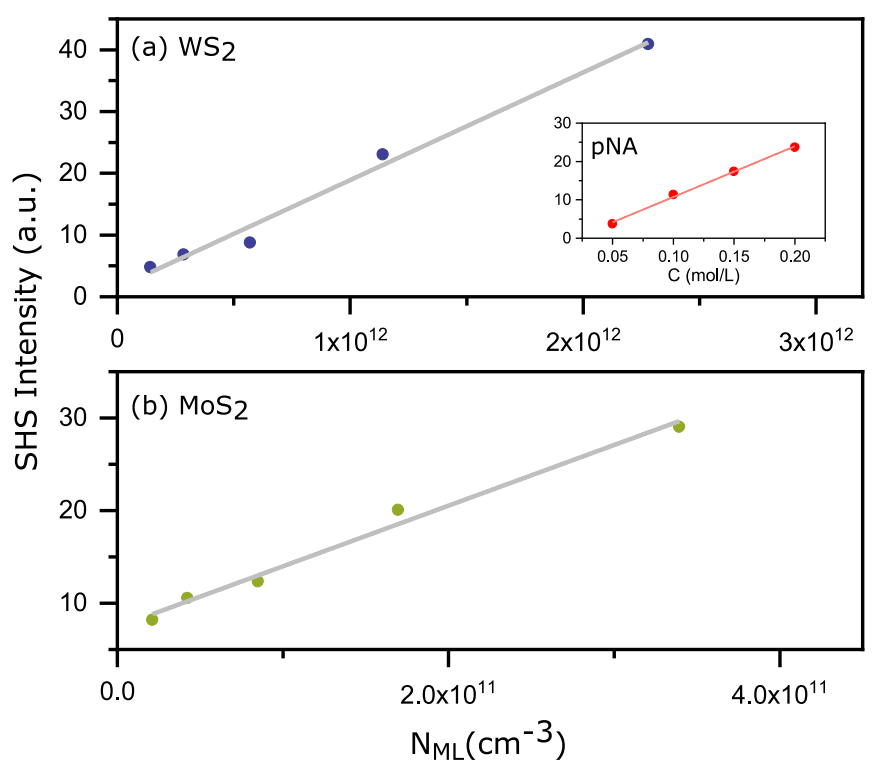

FIG. 3. Concentration-dependent SH signals at $\lambda_{\text {exc }}=1064 \mathrm{~nm}$ for (a) $\mathrm{WS}_{2}$ (sample w2; see Table I) and pNA and (b) $\mathrm{MoS}_{2} . N_{\mathrm{ML}}$ is the number density of monolayers, and $C$ is the molar concentration of pNA in methanol.

each excitation wavelength, emission spectra show a dominant SH signal together with two weak peaks. A background signal also appeared with unknown origin, but persisted across multiple $\mathrm{MoS}_{2}$ samples at varying concentrations, sizes, and syntheses. In any case, it was straightforward to deconvolve the SHS contribution from these spurious signals such that Supplemental Fig. S3(b) displays the ratio $I_{\mathrm{MoS}_{2}} / I_{\mathrm{LN}}$ resulting from these measurements.

Absolute measurements of $\chi^{(2)}$ are then performed at $1064 \mathrm{~nm}$ from the linear increase in SH intensities versus TMD (and pNA) concentrations, as described in Ref. [22]. From the measured slopes in Fig. 3, the TMD hyperpolarizability $\langle\beta\rangle$ can be calculated according to the standard formalism detailed in the Supplemental Material [7]. For TMDs, we remind the reader that only an odd number of layers can generate a SH signal and that, in a first-step approximation, odd layers all generate the same SH signal [11]. For each sample of Table I, typical layer distributions [26] show that the fraction of nanosheets with at least three layers is less than $10 \%$. We thus assume that the SH signal from high-order odd layers is negligible and that the relevant number concentration is the monolayer number concentration $N_{\mathrm{ML}}$. This parameter can be calculated from the weight concentration and monolayer volume fraction (mVf; see Supplemental Material [7]). The resulting $\chi^{(2)}$ values for $\mathrm{WS}_{2}$ and $\mathrm{MoS}_{2}$ are presented in Table I. For $\mathrm{WS}_{2}$, the measurements were performed on three independent suspensions with different monolayer volume fractions and size metrics. We obtained high $\chi_{x x x}^{(2)}$ values at $1064 \mathrm{~nm}$ varying between 270 and $530 \mathrm{pm} / \mathrm{V}$. For $\mathrm{MoS}_{2}, \chi_{x x x}^{(2)}$ was derived to be $130 \mathrm{pm} / \mathrm{V}$.

The benefit of the above approach is to propose an alternative method to SHG microscopy experiments, which is based here on ensemble measurements as already validated with other nanomaterials [34] and 2D sheets at $800 \mathrm{~nm}$ [39]. One major limitation for absolute measurements can be 


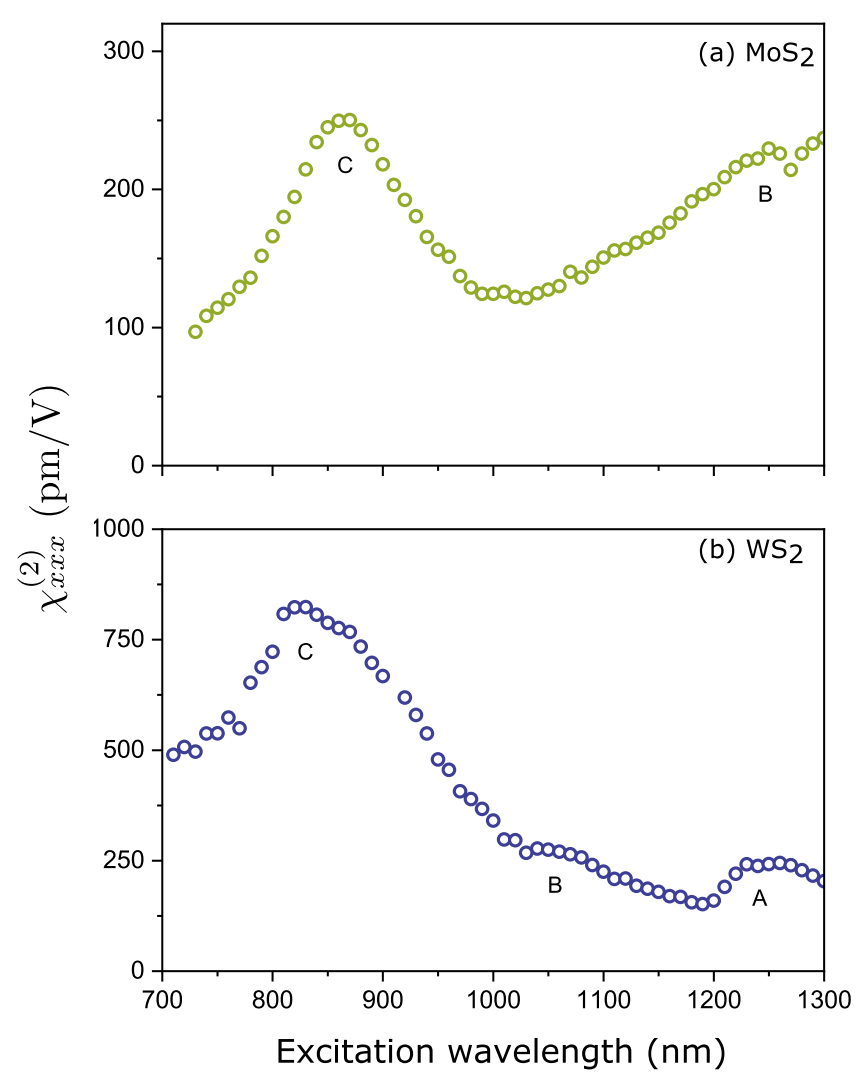

FIG. 4. Absolute nonlinear susceptibility $\chi_{x x x}^{(2)}$ of (a) $\mathrm{MoS}_{2}$ and (b) $\mathrm{WS}_{2}$ (sample w2; see Table I).

related, though, to the dispersion of the sample characteristics (nanosheet dimensions, distribution of the layer number), which is the reason why we used several suspensions of known metrics and with the highest possible monolayer content. The main uncertainty originates from the surface area estimation. According to TEM images, a rectangular shape with an average aspect ratio of 2 (i.e., $\langle S\rangle=\langle L\rangle^{2} / 2$ ) is usually retained [25]. Although uncertainty in $L$ results in negligible change in the calculated susceptibility [22], deviations in ideal rectangular shape at the same $L$ result in uncertainties of ca. $\pm 40 \%$ in the calculated susceptibilities when the aspect ratio is arbitrarily changed from 1 to 4 while keeping $L$ constant. If consistent $\chi^{(2)}$ values are thus obtained in Table I between the three different $\mathrm{WS}_{2}$ samples, we point out that only a further reduction in the nanosheet polydispersity can result at this stage in better quantitative assessments.

Finally, both relative measurements and absolute data at $1064 \mathrm{~nm}$ were combined to calculate the dispersion of the absolute $\chi^{(2)}$ over the excitation range as illustrated in Fig. 4. As previously discussed, our protocol allows us to account for the setup spectral response with high precision and reproducibility. We have, moreover, investigated the possible influence of the monolayer volume fraction on the spectral response of $\chi^{(2)}$. In Supplemental Fig. S2, the relative measures found for $\mathrm{WS}_{2}$ samples $\mathrm{w} 2$ and $\mathrm{w} 3$ are very similar; only a slightly higher resonance at $830 \mathrm{~nm}$ is observed for sample w3 with the highest proportion of monolayers. The absence of any wavelength shift [19] is to be mentioned, suggesting that the $\mathrm{SH}$ response within suspensions mainly originates from monolayers. However, we cannot exclude a contribution of the flake edges to the measured composite $\chi^{(2)}$ susceptibility for these nanosheets with low lateral dimensions. It was demonstrated that the SHG response could be modulated by the edge-type termination of the nanosheets, resulting in appearance of a resonance near 1300-nm excitation wavelength and higher overall magnitude [40,41]. The spectral responses shown in Fig. 4 are thus representative of TMD monolayers but may deviate from those typically obtained in SHG microscopy from large flakes obtained by CVD.

The as-derived $\chi^{(2)}$ dispersions for $\mathrm{WS}_{2}$ and $\mathrm{MoS}_{2}$ reveal resonances corresponding to the $\mathrm{A}, \mathrm{B}$, and $\mathrm{C}$ excitonic transitions in their respective electronic band structures. For $\mathrm{MoS}_{2}$, our data show susceptibility values at about $250 \mathrm{pm} / \mathrm{V}$ near the main $C$ excitonic resonance at $\lambda_{\text {exc }} \sim 870 \mathrm{~nm}$, corresponding well with the high-energy shoulder of the $\mathrm{C}$ transition resonance at $\sim 430 \mathrm{~nm}$ [see Fig. 1(a)]. For $\mathrm{WS}_{2}$, we found a generally higher susceptibility than for $\mathrm{MoS}_{2}$ with $\chi^{(2)}$ $\sim 820 \mathrm{pm} / \mathrm{V}$ around the $\mathrm{C}$ transition at $\lambda_{\text {exc }} \sim 830 \mathrm{~nm}$. In the linear regime, the $\mathrm{C}$ transition arises from a van Hove singularity where bands are parallel between the " $\Gamma$ " and " $\Lambda$ " points (commonly called the "nesting" region $[42,43]$ ), which provides a large density of states likely responsible for the measurably high $\chi^{(2)}$ magnitude in this region. In the 1000-1300-nm excitation range, our measurements evidence $\chi^{(2)}$ correlation with direct transitions across the " $K$ " point of the Brillouin zone. These are referred to as the A and B excitonic transitions for 2D TMDs in the linear regime but differ in energy due to spin-split valence bands. Both the two-photon A and B "features" were observable for $\mathrm{WS}_{2}$ (at $\sim 1230$ and $\sim 1050 \mathrm{~nm}$, respectively), but excitation of the $\mathrm{MoS}_{2} \mathrm{~A}$ feature was beyond the excitation capabilities of this setup (occurring beyond $1300 \mathrm{~nm}$ ).

One interest of this study is to measure on the same experimental setup and over a wide wavelength range the susceptibility of the two most studied TMD materials, $\mathrm{MoS}_{2}$ and $\mathrm{WS}_{2}$, for a direct comparison with literature values. Available data showing the dispersion of the $\chi^{(2)}$ have been normalized and plotted together in Supplemental Fig. S4. These data evidence a strong $\mathrm{C}$ exciton resonance in general agreement with our measurements. Comparison with discrete absolute literature values (Supplemental Table S1 and Supplemental Fig. S5) is more problematic due to their large discrepancy, as already introduced. For $\mathrm{MoS}_{2}$, our data are in good agreement with pioneer works [3,4] and a very recent work [9] demonstrating susceptibility values at about several hundreds of $\mathrm{pm} / \mathrm{V}$ near the main $\mathrm{C}$ excitonic resonance. In the 1100 1200-nm excitation range, it should be pointed out that lower values have been measured $(30 \mathrm{pm} / \mathrm{V}$ near the $\mathrm{A}$ transition [8]), whereas susceptibilities at only a few $\mathrm{pm} / \mathrm{V}$ were obtained under off-resonant conditions at $1560 \mathrm{~nm}[11,12]$. These values clearly contradict our measurements, but we point out that our $\chi^{(2)}$ dispersion spectra allow us to connect the various spectral regions that were separately studied in the literature. If uncertainty of $\pm 40 \%$ is here proposed for $\chi^{(2)}$ absolute measurements, our protocol allows us to retrieve the relative dispersion with good accuracy. Our $\chi^{(2)}$ values are significantly higher at the A resonance and probably off-resonance in comparison to those measured in previous studies, possibly attributable to the ostensible contribution of 
edge effects as previously discussed. It is, however, difficult to better assess this contribution because of the nanosheet preparation from liquid-cascade centrifugation, which shows a high monolayer concentration but inherently small lateral dimensions. For $\mathrm{WS}_{2}$, less work has been performed, and a lower susceptibility (275 pm/V) was found in Ref. [9] near the $\mathrm{C}$ resonance. In addition, it can be noted that theoretical calculations $[20,44]$ yielded to high values $(\sim 1000 \mathrm{pm} / \mathrm{V})$ for the nonlinear susceptibility of both $\mathrm{MoS}_{2}$ and $\mathrm{WS}_{2}$ in this excitation range.

\section{CONCLUSION}

Compared with the available literature data, an alternative experimental approach is here used to retrieve in a broader excitation range the $\chi^{(2)}$ dispersion of both $\mathrm{MoS}_{2}$ and $\mathrm{WS}_{2}$ nanosheets. From monodisperse and well-characterized liquid-exfoliated TMD suspensions showing a high mono- layer content and low lateral dimensions, we show that accurate $\chi^{(2)}$ values can be obtained with SHS spectroscopy with uncertainties estimated at $\pm 40 \%$ arising from sample polydispersity inherent to both ensemble measurements and liquid exfoliation preparation. $\chi^{(2)}$ dispersion measurements show high susceptibility values over the whole spectral range and clear resonances associated with the main A, B, and C excitonic transitions.

\section{ACKNOWLEDGMENTS}

The authors acknowledge financial support from the France-Switzerland Interreg V program (project OncoNanoScreen), from the Agence Nationale de la Recherche (project RACINE), from the 2015-2020 French Contrat de Plan ÉtatRégion (project E-TIME) and from the NSWC-Crane NISE 219 program.
[1] A. Autere, H. Jussila, Y. Dai, Y. Wang, H. Lipsanen, and Z. Sun, Nonlinear optics with 2D layered materials, Adv. Mater. 30, 1705963 (2018).

[2] Y. Wang, J. Xiao, S. Yang, Y. Wang, and X. Zhang, Second harmonic generation spectroscopy on two-dimensional materials [invited], Opt. Mater. Express 9, 1136 (2019).

[3] L. M. Malard, T. V. Alencar, A. P. M. Barboza, K. F. Mak, and A. M. de Paula, Observation of intense second harmonic generation from $\mathrm{MoS}_{2}$ atomic crystals, Phys. Rev. B 87, 201401(R) (2013)

[4] Y. Li, Y. Rao, K. F. Mak, Y. You, S. Wang, C. R. Dean, and T. F. Heinz, Probing symmetry properties of few-layer $\mathrm{MoS}_{2}$ and h-BN by optical second-harmonic generation, Nano Lett. 13, 3329 (2013).

[5] W.-T. Hsu, Z.-A. Zhao, L.-J. Li, C.-H. Chen, M.-H. Chiu, P.-S. Chang, Y.-C. Chou, and W.-H. Chang, Second harmonic generation from artificially stacked transition metal dichalcogenide twisted bilayers, ACS Nano 8, 2951 (2014).

[6] M. L. Trolle, Y.-C. Tsao, K. Pedersen, and T. G. Pedersen, Observation of excitonic resonances in the second harmonic spectrum of $\mathrm{MoS}_{2}$, Phys. Rev. B 92, 161409(R) (2015).

[7] See Supplemental Material at http://link.aps.org/supplemental/ 10.1103/PhysRevB.102.235408 for a description of the sample preparation, the derivation of the absolute susceptibility at $1064 \mathrm{~nm}$, a comparison of the $\chi^{(2)}$ relative dispersion for $\mathrm{WS}_{2}$ suspensions, the $\mathrm{MoS}_{2}$ data, and a comparison with the literature data.

[8] D. J. Clark, C. T. Le, V. Senthilkumar, F. Ullah, H.-Y. Cho, Y. Sim, M.-J. Seong, K.-H. Chung, Y. S. Kim, and J. I. Jang, Near bandgap second-order nonlinear optical characteristics of $\mathrm{MoS}_{2}$ monolayer transferred on transparent substrates, Appl. Phys. Lett. 107, 131113 (2015).

[9] K. Yao, E. Yanev, H.-J. Chuang, M. R. Rosenberger, X. Xu, T. Darlington, K. M. McCreary, A. T. Hanbicki, K. Watanabe, T. Taniguchi, B. T. Jonker, X. Zhu, D. N. Basov, J. C. Hone, and P. J. Schuck, Continuous wave sum frequency generation and imaging of monolayer and heterobilayer two-dimensional semiconductors, ACS Nano 14, 708 (2020).
[10] R. I. Woodward, R. T. Murray, C. F. Phelan, R. E. P. de Oliveira, T. H. Runcorn, E. J. R. Kelleher, S. Li, E. C. de Oliveira, G. J. M. Fechine, G. Eda, and C. J. S. de Matos, Characterization of the second- and third-order nonlinear optical susceptibilities of monolayer $\mathrm{MoS}_{2}$ using multiphoton microscopy, 2D Mater. 4, 011006 (2016).

[11] A. Säynätjoki, L. Karvonen, H. Rostami, A. Autere, S. Mehravar, A. Lombardo, R. A. Norwood, T. Hasan, N. Peyghambarian, H. Lipsanen, K. Kieu, A. C. Ferrari, M. Polini, and Z. Sun, Ultra-strong nonlinear optical processes and trigonal warping in $\mathrm{MoS}_{2}$ layers, Nat. Commun. 8, 893 (2017).

[12] A. Autere, H. Jussila, A. Marini, J. R. M. Saavedra, Y. Dai, A. Säynätjoki, L. Karvonen, H. Yang, B. Amirsolaimani, R. A. Norwood, N. Peyghambarian, H. Lipsanen, K. Kieu, F. J. G. de Abajo, and Z. Sun, Optical harmonic generation in monolayer group-VI transition metal dichalcogenides, Phys. Rev. B 98, 115426 (2018).

[13] X. Miao, N. Xuan, Q. Liu, W. Wu, H. Liu, Z. Sun, and M. $\mathrm{Ji}$, Optimizing nonlinear optical visibility of two-dimensional materials, ACS Appl. Mater. Interfaces 9, 34448 (2017).

[14] J. Liang, J. Zhang, Z. Li, H. Hong, J. Wang, Z. Zhang, X. Zhou, R. Qiao, J. Xu, P. Gao, Z. Liu, Z. Liu, Z. Sun, S. Meng, K. Liu, and D. Yu, Monitoring local strain vector in atomic-layered $\mathrm{MoSe}_{2}$ by second-harmonic generation, Nano Lett. 17, 7539 (2017).

[15] H. Li, J. Wu, Z. Yin, and H. Zhang, Preparation and applications of mechanically exfoliated single-layer and multilayer $\mathrm{MoS}_{2}$ and $\mathrm{WSe}_{2}$ nanosheets, Acc. Chem. Res. 47, 1067 (2014).

[16] C. Janisch, Y. Wang, D. Ma, N. Mehta, A. L. Elías, N. PereaLópez, M. Terrones, V. Crespi, and Z. Liu, Extraordinary second harmonic generation in tungsten disulfide monolayers, Sci. Rep. 4, 5530 (2014).

[17] M. Mokim, A. Card, and F. Ganikhanov, Nonlinear optical susceptibility of atomically thin $\mathrm{W} X_{2}$ crystals, Opt. Mater. (Amsterdam, Neth.) 88, 30 (2019).

[18] D. J. Clark, V. Senthilkumar, C. T. Le, D. L. Weerawarne, B. Shim, J. I. Jang, J. H. Shim, J. Cho, Y. Sim, M.-J. Seong, S. H. Rhim, A. J. Freeman, K.-H. Chung, and Y. S. Kim, Strong 
optical nonlinearity of CVD-grown $\mathrm{MoS}_{2}$ monolayer as probed by wavelength-dependent second-harmonic generation, Phys. Rev. B 90, 121409(R) (2014).

[19] Y. Kikuchi, Y. Miyauchi, R. Takaoka, T. Suzuki, M. Tanaka, and S. Ohno, Multiple-peak resonance of optical second harmonic generation arising from band nesting in monolayer transition metal dichalcogenides $T X_{2}$ on $\mathrm{SiO}_{2} / \mathrm{Si}(001)$ substrates $(T=$ Mo, W; $X=\mathrm{S}$, Se), Phys. Rev. B 100, 075301 (2019).

[20] G. F. Mkrtchian, A. Knorr, and M. Selig, Theory of secondorder excitonic nonlinearities in transition metal dichalcogenides, Phys. Rev. B 100, 125401 (2019).

[21] M. C. Lucking, K. Beach, and H. Terrones, Large second harmonic generation in alloyed TMDs and boron nitride nanostructures, Sci. Rep. 8, 10118 (2018).

[22] G. T. Forcherio, J. Riporto, J. R. Dunklin, Y. Mugnier, R. L. Dantec, L. Bonacina, and D. K. Roper, Nonlinear optical susceptibility of two-dimensional $\mathrm{WS}_{2}$ measured by hyper Rayleigh scattering, Opt. Lett. 42, 5018 (2017); 43, 2400(E) (2018).

[23] J. N. Coleman, M. Lotya, A. O’Neill, S. D. Bergin, P. J. King, U. Khan, K. Young, A. Gaucher, S. De, R. J. Smith, I. V. Shvets, S. K. Arora, G. Stanton, H.-Y. Kim, K. Lee, G. T. Kim, G. S. Duesberg, T. Hallam, J. J. Boland, J. J. Wang et al., Two-dimensional nanosheets produced by liquid exfoliation of layered materials, Science 331, 568 (2011).

[24] C. Backes, B. M. Szydłowska, A. Harvey, S. Yuan, V. VegaMayoral, B. R. Davies, P.-1. Zhao, D. Hanlon, E. J. G. Santos, M. I. Katsnelson, W. J. Blau, C. Gadermaier, and J. N. Coleman, Production of highly monolayer enriched dispersions of liquidexfoliated nanosheets by liquid cascade centrifugation, ACS Nano 10, 1589 (2016).

[25] C. Backes, R. J. Smith, N. McEvoy, N. C. Berner, D. McCloskey, H. C. Nerl, A. O’Neill, P. J. King, T. Higgins, D. Hanlon, N. Scheuschner, J. Maultzsch, L. Houben, G. S. Duesberg, J. F. Donegan, V. Nicolosi, and J. N. Coleman, Edge and confinement effects allow in situ measurement of size and thickness of liquid-exfoliated nanosheets, Nat. Commun. 5, 4576 (2014).

[26] L. Ueberricke, J. N. Coleman, and C. Backes, Robustness of size selection and spectroscopic size, thickness and monolayer metrics of liquid-exfoliated $\mathrm{WS}_{2}$, Phys. Status Solidi B 254, 1700443 (2017).

[27] M. Lotya, A. Rakovich, J. F. Donegan, and J. N. Coleman, Measuring the lateral size of liquid-exfoliated nanosheets with dynamic light scattering, Nanotechnology 24, 265703 (2013).

[28] K. Synnatschke, P. A. Cieslik, A. Harvey, A. CastellanosGomez, T. Tian, C.-J. Shih, A. Chernikov, E. J. G. Santos, J. N. Coleman, and C. Backes, Length- and thickness-dependent optical response of liquid-exfoliated transition metal dichalcogenides, Chem. Mater. 31, 10049 (2019).

[29] C. Backes, D. Campi, B. M. Szydlowska, K. Synnatschke, E. Ojala, F. Rashvand, A. Harvey, A. Griffin, Z. Sofer, N. Marzari, J. N. Coleman, and D. D. O'Regan, Equipartition of energy defines the size-thickness relationship in liquidexfoliated nanosheets, ACS Nano 13, 7050 (2019).

[30] J. R. Dunklin, P. Lafargue, T. M. Higgins, G. T. Forcherio, M. Benamara, N. McEvoy, D. K. Roper, J. N. Coleman, Y.
Vaynzof, and C. Backes, Production of monolayer-rich golddecorated $2 \mathrm{H}-\mathrm{WS}_{2}$ nanosheets by defect engineering, npj 2D Mater. Appl. 1, 43 (2017).

[31] S. P. Ogilvie, M. J. Large, M. A. O’Mara, P. J. Lynch, C. L. Lee, A. A. K. King, C. Backes, and A. B. Dalton, Size selection of liquid-exfoliated 2D nanosheets, 2D Mater. 6, 031002 (2019).

[32] A. Harvey, C. Backes, J. B. Boland, X. He, A. Griffin, B. Szydlowska, C. Gabbett, J. F. Donegan, and J. N. Coleman, Non-resonant light scattering in dispersions of 2D nanosheets, Nat. Commun. 9, 4553 (2018).

[33] A. Griffin, A. Harvey, B. Cunningham, D. Scullion, T. Tian, C.J. Shih, M. Gruening, J. F. Donegan, E. J. G. Santos, C. Backes, and J. N. Coleman, Spectroscopic size and thickness metrics for liquid-exfoliated h-BN, Chem. Mater. 30, 1998 (2018).

[34] J. Riporto, M. Urbain, Y. Mugnier, V. Multian, F. Riporto, K. Bredillet, S. Beauquis, C. Galez, V. Monnier, Y. Chevolot, V. Gayvoronsky, L. Bonacina, and R. Le Dantec, Second harmonic spectroscopy of $\mathrm{ZnO}, \mathrm{BiFeO}_{3}$ and $\mathrm{LiNbO}_{3}$ nanocrystals, Opt. Mater. Express 9, 1955 (2019).

[35] F. L. Huyskens, P. L. Huyskens, and A. P. Persoons, Solvent dependence of the first hyperpolarizability of $p$-nitroanilines: Differences between nonspecific dipole-dipole interactions and solute-solvent H-bonds, J. Chem. Phys. 108, 8161 (1998).

[36] R. Le Dantec, Y. Mugnier, G. Djanta, L. Bonacina, J. Extermann, L. Badie, C. Joulaud, M. Gerrmann, D. Rytz, J. P. Wolf, and C. Galez, Ensemble and individual characterization of the nonlinear optical properties of $\mathrm{ZnO}$ and $\mathrm{BaTiO}_{3}$ nanocrystals, J. Phys. Chem. C 115, 15140 (2011).

[37] Y. R. Shen, Optical second harmonic generation at interfaces, Annu. Rev. Phys. Chem. 40, 327 (1989).

[38] J. Campo, F. Desmet, W. Wenseleers, and E. Goovaerts, Highly sensitive setup for tunable wavelength hyper-Rayleigh scattering with parallel detection and calibration data for various solvents, Opt. Express 17, 4587 (2009).

[39] I. Russier-Antoine, H. Fakhouri, S. Basu, F. Bertorelle, P. Dugourd, P.-F. Brevet, P. Velayudhan, S. Thomas, N. Kalarikkal, and R. Antoine, Second harmonic scattering from mass characterized 2D graphene oxide sheets, Chem. Commun. (Cambridge, U. K.) 56, 3859 (2020).

[40] X. Yin, Z. Ye, D. A. Chenet, Y. Ye, K. O’Brien, J. C. Hone, and $\mathrm{X}$. Zhang, Edge nonlinear optics on a $\mathrm{MoS}_{2}$ atomic monolayer, Science 344, 488 (2014).

[41] K.-I. Lin, Y.-H. Ho, S.-B. Liu, J.-J. Ciou, B.-T. Huang, C. Chen, H.-C. Chang, C.-L. Tu, and C.-H. Chen, Atom-dependent edgeenhanced second-harmonic generation on $\mathrm{MoS}_{2}$ monolayers, Nano Lett. 18, 793 (2018).

[42] D. Kozawa, R. Kumar, A. Carvalho, K. Kumar Amara, W. Zhao, S. Wang, M. Toh, R. M. Ribeiro, A. H. Castro Neto, K. Matsuda, and G. Eda, Photocarrier relaxation pathway in twodimensional semiconducting transition metal dichalcogenides, Nat. Commun. 5, 4543 (2014).

[43] D. Y. Qiu, F. H. da Jornada, and S. G. Louie, Optical Spectrum of $\mathrm{MoS}_{2}$ : Many-Body Effects and Diversity of Exciton States, Phys. Rev. Lett. 111, 216805 (2013).

[44] M. L. Trolle, G. Seifert, and T. G. Pedersen, Theory of excitonic second-harmonic generation in monolayer $\mathrm{MoS}_{2}$, Phys. Rev. B 89, 235410 (2014). 